\title{
INTEGRACIÓN DEL ÍNDICE DE VEGETACIÓN DE LA DIFERENCIA NORMALIZADA (NDVI) Y DEL CICLO FENOLÓGICO DE MAÍZ PARA ESTIMAR EL RENDIMIENTO A ESCALA DEPARTAMENTAL EN CÓRDOBA, ARGENTINA
}

\author{
Normalized Difference Vegetation Index (NDVI) and phenological data integration to \\ estimate county yield of corn in Córdoba, Argentina
}

\author{
Antonio de la Casa ${ }^{1}$, y Gustavo Ovando ${ }^{1}$
}

\section{A B S T R A C T}

Integration of satellite data with agroclimatic information can result in better procedures to evaluate the state and evolution of grain crops. In this work, phenologic calendar of corn (Zea mays L.) crop based on growing-degree days procedures was integrated with Normalized Difference Vegetation Index (NDVI) estimations from global coverage of National Oceanic and Atmospheric Administration-Advanced Very High Resolution Radiometer (NOAA-AVHRR) system. The main objectives were: i) to evaluate the relationship between NDVI and corn yield in different stages of crop cycle; ii) to analyze the influence of sowing date, and iii) to develop a predictive model of county (departmental) corn yield using satellite and ground data. The NDVI values accumulated in different corn phenologic stages showed a positive association with yield, and this relationship was modified in function of sowing date. The NDVI value during the reproductive stage, for any sowing date, always expressed a high association with corn yield, reaching significant correlation values $(\mathrm{P}<0.05)$ in all cases, and even higher $(\mathrm{P}<0.01)$ for some evaluated dates. The higher sensitivity showed by the reproductive stage confirms that it is a critical period. Starting from this information, a prediction model was obtained that explains around $80 \%$ of corn yield variability of Marcos Juárez Department in Córdoba Province, Argentina.

Key words: corn, yield, development stages, sowing date, Zea mays L.

\section{R E S U M E N}

La integración de datos radiométricos con información agroclimática puede resultar en mejores procedimientos para evaluar el estado y evolución de los cultivos. En este trabajo, datos del calendario fenológico del cultivo de maíz (Zea mays L.), estimados a partir de procedimientos de sumas térmicas, fueron integrados con registros del Índice de Vegetación de la Diferencia Normalizada (NDVI) de cobertura global del sistema Radiómetro Avanzado de Muy Alta Resolución de la Administración Nacional del Océano y la Atmósfera de los Estados Unidos (NOAAAVHRR), con los siguientes objetivos: i) evaluar la relación entre el NDVI y el rendimiento de maíz en distintas etapas del ciclo del cultivo; ii) analizar la influencia de la fecha de siembra, y iii) desarrollar un modelo de pronóstico del rendimiento de maíz a escala departamental. Los valores acumulados de NDVI presentaron una asociación positiva con el rendimiento de maíz, variable de acuerdo a la etapa de desarrollo y la fecha de siembra consideradas. Durante la etapa reproductiva, el NDVI expresó una elevada asociación con el rendimiento en cualquier fecha de siembra eventual, alcanzando valores de correlación significativos $(\mathrm{P}<0,05)$ en todos los casos, y aún superiores $(\mathrm{P}<0,01)$ en algunas de las fechas evaluadas. La mayor sensibilidad que presenta la etapa reproductiva del cultivo de maíz pone de manifiesto su condición de período crítico. A partir de esta información, se obtuvo un modelo de predicción que explica alrededor del $80 \%$ de la variabilidad del rendimiento de maíz del Departamento Marcos Juárez en la Provincia de Córdoba, Argentina.

Palabras clave: maíz, rendimiento, etapas de desarrollo, fechas de siembra, Zea mays L.

\footnotetext{
${ }^{1}$ Universidad Nacional de Córdoba, Facultad de Ciencias Agropecuarias, CC 509-Ciudad Universitaria, 5000 Córdoba, Argentina. E-mail: delacasa@agro.uncor.edu *Autor para correspondencia.

Recibido: 19 de diciembre de 2006. Aceptado: 5 de abril de 2007.
} 


\section{INTRODUCCIÓN}

La predicción del rendimiento de los cultivos anticipada a la cosecha ha sido un objetivo prioritario de la agrometeorología, tanto con el fin de aumentar el conocimiento de las relaciones clima/ cultivo como para generar información que se pueda aprovechar oportunamente en la planificación y manejo de la producción agropecuaria. Las técnicas de estimación del rendimiento se incrementaron con el desarrollo de la tecnología satelital, dando lugar a una gama de posibilidades que comprenden, además de los procedimientos originales en base a modelos matemáticos de simulación de cultivos, a los que emplean información exclusivamente satelital, como así también los que integran ambas fuentes de datos (Weiss et al., 2001; Doraiswamy et al., 2003).

La oficina que elabora las estadísticas de la producción agrícola en la Provincia de Córdoba utiliza procedimientos tradicionales, basados en encuestas para establecer el área de siembra, cosecha, rendimiento y producción de los cultivos de mayor importancia económica de la región (SAGyA, 2002). Esta información se elabora a nivel departamental y, por lo general, hace las veces de variable dependiente en los modelos regionales de estimación del rendimiento (de la Casa, 1992). La estimación del rendimiento de cultivos en áreas extensas, que se obtiene tanto a través de encuestas como mediante la utilización de modelos de cultivo, adolece de algunos defectos como son: la imprecisión, que deriva de una cobertura espacial limitada del procedimiento; y la oportunidad, dado que la información de rendimiento y producción se obtiene en una fecha posterior a la cosecha (Dawbin et al., 1980).

La ventaja de emplear datos satelitales para el pronóstico del rendimiento agrícola es su capacidad de observación global del territorio, aunque esta tecnología también tiene puntos débiles y restricciones. Así, en una primera etapa de su aplicación para el seguimiento de la condición de los cultivos fueron empleadas imágenes del satélite LANDSAT, que posee una resolución temporal limitada de aproximadamente 16 días. La baja frecuencia de las observaciones (por su propio funcionamiento y la ocurrencia de nubosidad) representa una restricción operacional importante, que procuró remediarse acoplando los datos de origen satelital con la in- formación derivada de modelos de cultivos. Por lo general, los modelos de cultivos proveen estimaciones diarias de su evolución (Maas, 1988; 1993), lo que permite salvar la discontinuidad propia de la información satelital con mayor resolución espacial.

La capacidad de observación del territorio se amplió con la utilización de sistemas satélite-sensor de mayor resolución temporal como el Radiómetro Avanzado de Muy Alta Resolución de la Administración Nacional del Océano y la Atmósfera de los Estados Unidos (NOAA-AVHRR), cuyos datos tienen una frecuencia diaria y son de menor costo que las imágenes LANDSAT, aunque su resolución espacial es de menor detalle, aproximadamente 100 ha (Smith et al., 1995). El proyecto Pathfinder sistematizó e integró una base de datos del Índice de Vegetación de la Diferencia Normalizada (NDVI) a escala global, que en la actualidad permite disponer de una serie prolongada de registros del NDVI. Estos datos son apropiados para evaluar la variabilidad interanual de las series de rendimiento agrícola agregados por unidad territorial administrativa, como fuera realizado por Mika et al. (2002) para los cultivos de trigo (Triticum aestivum L.) y maíz en Hungría.

Chang et al. (2003) han establecido que los modelos de pronóstico del rendimiento de maíz desarrollados a partir de información radiométrica explican una mayor proporción de la variabilidad del rendimiento, por lo general, cuando consideran varias fechas de muestreo durante el ciclo. Por su parte, Shanahan et al. (2001) determinaron que el uso del Índice de Vegetación de la Diferencia Normalizada Verde (GNDVI), una variante del NDVI que utiliza la información del canal verde en lugar del rojo, presentó una fuerte correlación con el rendimiento de maíz, en particular durante la etapa media de llenado del grano.

Los primeros estudios conducidos a escala de predio han mostrado que los datos obtenidos a partir de sensores remotos pueden ser incorporados en los modelos de simulación de cultivo para calibrar o ajustar sus parámetros durante el período de simulación, a fin de asegurar que la condición simulada del crecimiento, desarrollo o rendimiento del cultivo, concuerde con la observada (Doraiswamy et al., 2003). 
Los modelos de simulación de cultivos estiman la tasa y duración de los procesos de crecimiento y desarrollo, recurriendo a procedimientos basados en sumas térmicas o grados días. Estas técnicas determinan en forma simple el momento de ocurrencia de un evento fenológico a partir de información térmica, superando la mayor inexactitud que deriva de usar los días calendarios de manera directa (Warrington y Kanemasu, 1983; Kiniry y Bonhomme, 1991; Stewart et al., 1998). Esta información es relevante porque las etapas de desarrollo de los cultivos presentan condiciones de sensibilidad particular frente al estrés hídrico. En el caso del maíz, la floración es una fase decisiva en la determinación del rendimiento final, razón por la cual las condiciones climáticas que acompañan su ocurrencia son utilizadas de manera particular con fines de pronóstico (Dale y Daniels, 1995) o para evaluar el impacto de la sequía (Meyer et al., 1993a; 1993b). También la información radiométrica, en virtud de la interacción de la energía luminosa con la canopia del cultivo, resulta particularmente dependiente de la etapa fenológica durante el ciclo. Así, Lobell et al. (2003) utilizan una función de suma térmica para modelar la variación que experimenta la radiación fotosintéticamente activa absorbida por la canopia durante el ciclo del trigo.

Por su parte, existen distintos niveles de complejidad e integración de los datos de satélite en los modelos de cultivos (Maas, 1988; Weiss et al., 2001; Doraiswamy et al., 2003). En este trabajo se propone integrar datos del calendario fenológico del cultivo de maíz, estimados a partir del método de sumas térmicas, con datos de NDVI que fueron obtenidos por el sistema NOAA-AVHRR y están disponibles en un formato de cobertura global. Este análisis ha sido acotado geográficamente al sector comprendido por el Departamento Marcos Juárez, que constituye una de las principales áreas de producción de maíz en la Provincia de Córdoba.

Dado que no existe un sistema de pronóstico de rendimiento de cultivos en la Provincia de Córdoba, que esté basado en modelos de simulación o que haga uso de información radiométrica de origen satelital, los objetivos del trabajo fueron: 1) evaluar la relación entre el NDVI y el rendimiento de maíz en distintas etapas del ciclo del cultivo; 2) analizar la influencia de la fecha de siembra sobre estas relaciones; y 3) desarrollar un modelo de pronóstico del rendimiento departamental de maíz a partir de la información producida. Como paso previo a la obtención de un modelo de predicción de rendimiento, se requiere verificar el alcance local de la rutina de sumas térmicas para representar el calendario fenológico del maíz en la región.

\section{MATERIALES Y MÉTODOS}

\section{Datos de NDVI}

Los datos de NDVI fueron provistos por Goddard Space Flight Center (Greenbelt, Maryland, USA) de NOAA/NASA y corresponden al proyecto Pathfinder AVHRR Land (PAL) (Agbu y James, 1994). Las imágenes originales se presentan corregidas radiométricamente (la reflectancia de los canales 1 y 2 está corregida por la dispersión de Rayleigh y la absorción del ozono) y la proyección geográfica responde a la Homolosina Interrumpida de Goode, diseñada para reducir distorsiones de las principales masas continentales (Steinwand et al., 1992).

El NDVI se define como (Tarpley et al., 1984):

$N D V I=\frac{\rho_{\text {nir }}-\rho_{r}}{\rho_{\text {nir }}+\rho_{r}}$

donde $\rho_{\text {nir }}$ y $\rho_{r}$ son los valores de reflectancia correspondientes a las longitudes de onda del infrarrojo cercano y del rojo (canales 2 y 1 del NOAAAVHRR), respectivamente.

Las imágenes resultantes del procesamiento, que originalmente presentan un formato digital de 8 bits $[0,255](C D)$, se transformaron a la expresión real del NDVI $[-1,1]$ usando el valor correspondiente de ganancia $(0,008)$ y offset $(128)$, de acuerdo a la siguiente expresión (Agbu y James, 1994):

$$
N D V I=(C D-128) \times 0,008
$$

La serie disponible de imágenes de NDVI, compuestas en períodos de 10 días (Holben, 1986) y con formato de cobertura global (con un píxel de 8 $\mathrm{km}$ ), se inicia en julio de 1981 y se extiende hasta diciembre de 1999. En esta primera etapa del análisis se utilizaron las imágenes entre agosto de 1981 y mayo de 1991, para abarcar 11 ciclos consecutivos de maíz. El resto de las imágenes se reservó para efectuar una verificación posterior del modelo. Los datos de NDVI se ordenaron desde agosto de un año hasta mayo del siguiente, para evaluar el ciclo potencial de cultivo en cada campaña agrícola. 
La ubicación geográfica del Departamento Marcos Juárez en la Provincia de Córdoba, Argentina, se ubica en las coordenadas $32^{\circ} 03^{\prime}-33^{\circ} 56^{\prime}$ lat. Sur; $61^{\circ} 47^{\prime}-62^{\circ} 48^{\prime}$ long. Oeste. Por medio de la superposición del mapa vectorial, correspondiente a la localización geográfica de los límites políticos del Departamento Marcos Juárez, sobre cada imagen raster (matriz de datos) de la serie de NDVI decádico, se extrajo el conjunto de píxeles a fin de obtener los estadísticos descriptivos de esta región para la serie analizada.

\section{Datos de rendimiento de maíz}

La información estadística de la producción de maíz del Departamento Marcos Juárez fue provista por la dependencia encargada del gobierno de la Provincia de Córdoba (SAGyA, 2002).

\section{Datos de temperatura para evaluar la rutina de sumas térmicas}

Para realizar los cálculos de sumas térmicas se utilizó la información de la temperatura del aire de la estación meteorológica Marcos Juárez AERO (32 $42^{\prime}$ lat. Sur; $62^{\circ} 09^{\prime}$ long. Oeste). Se asumió que estos valores eran válidos para todo el departamento homónimo. Se dispuso de una serie de datos diarios de temperatura máxima $\left(\mathrm{T}^{\mathrm{o}} \max ,{ }^{\circ} \mathrm{C}\right)$ y de temperatura mínima $\left(\mathrm{T}^{\circ} \mathrm{min},{ }^{\circ} \mathrm{C}\right)$ entre 1968 y 1991, a los efectos de calcular las sumas de grados días (GD) con la siguiente expresión según la
Organización Meteorológica Mundial (WMO, 1983):

$G D=\left(\frac{T^{o} 08: 00 h+T^{o} 20: 00 h+T^{o} \max +T^{o} \min }{4}\right)-T b$

Si $\mathrm{T}^{\circ} \max >30^{\circ} \mathrm{C}$, entonces $\mathrm{T}^{\mathrm{o}} \max =30^{\circ} \mathrm{C} ; \mathrm{Si} \mathrm{T}^{\circ} \min$ $<10{ }^{\circ} \mathrm{C}$, entonces $\mathrm{T}^{\circ} \mathrm{min}=10^{\circ} \mathrm{C} ; \mathrm{Tb}=$ temperatura base $\left({ }^{\circ} \mathrm{C}\right)$.

Por su parte, $\mathrm{Tb}$ se estableció en $10{ }^{\circ} \mathrm{C}$ (Brown y Bootsma, 1993) y $8{ }^{\circ} \mathrm{C}$ (Ritchie y Nesmith, 1991), de acuerdo al requerimiento particular de los distintos métodos de sumas térmicas aplicados.

\section{Procedimientos de sumas térmicas}

Se utilizaron dos procedimientos de sumas térmicas. El primero para establecer el calendario fenológico del cultivo de maíz, de acuerdo al esquema de etapas de desarrollo o subperíodos propuesto en los modelos de Doorembos y Kassam (1979), como el utilizado por Barron et al. (2003) para analizar el efecto de los períodos secos sobre el rendimiento del maíz durante la estación de cultivo. Dicho esquema considera cuatro subperíodos entre siembra y cosecha: establecimiento, vegetativo, reproductivo y maduración, de acuerdo a la variación de los umbrales de GD acumulados $\left(\mathrm{Tb}=10^{\circ} \mathrm{C}\right)$, con respecto a la latitud geográfica (Burt et al., 1981), como muestra la Figura 1.

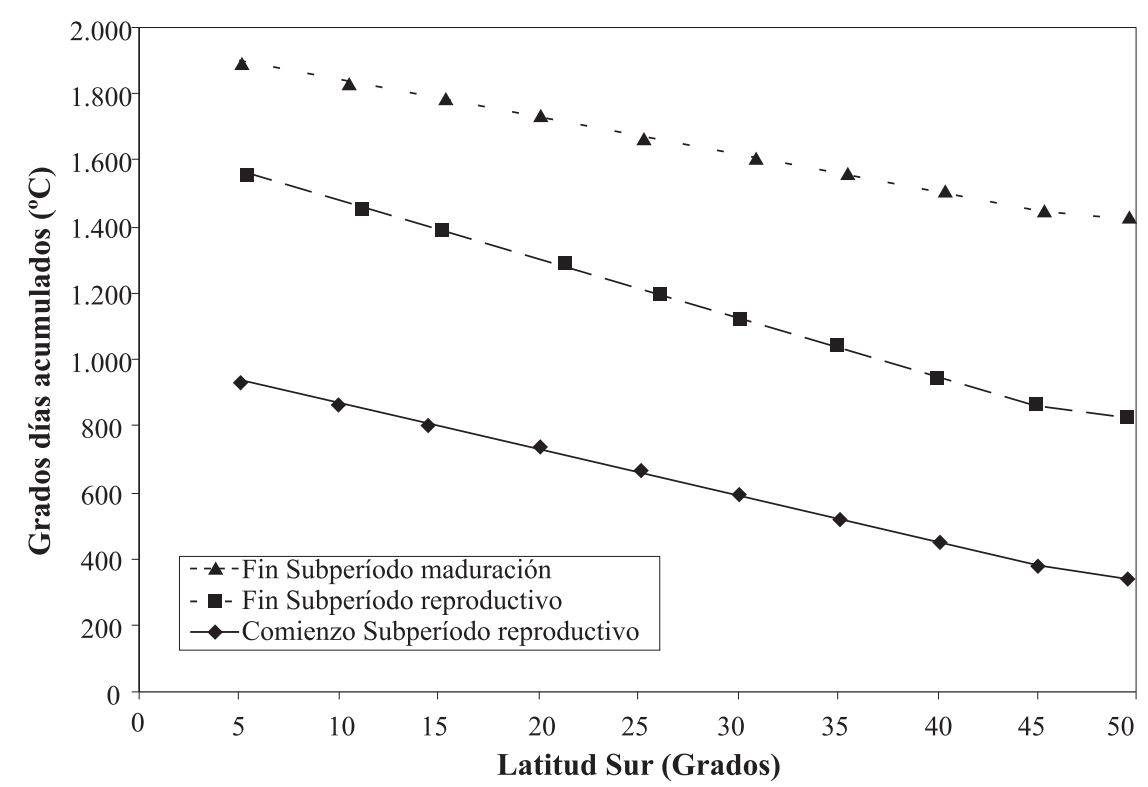

Figura 1. Tiempo térmico acumulado para estimar la duración de las etapas de desarrollo del maíz en función de la latitud (Burt et al., 1981).

Figure 1. Accumulated thermal time to estimate the length of corn development stages in relation to latitude (Burt et al., 1981). 
La etapa de establecimiento, que ocurre entre siembra y emergencia, se incluyó en el subperíodo vegetativo, asumiendo un requerimiento fijo de sumas térmicas de $66^{\circ} \mathrm{C}$ días (Nielsen et al., 2002). A los efectos de verificar el alcance local del procedimiento anterior, se utilizó información de un ensayo conducido en la Estación Experimental Agropecuaria Marcos Juárez del Instituto Nacional de Tecnología Agropecuaria, de acuerdo con la cual los cultivares de maíz de uso en la región requieren una acumulación de alrededor de $850 \mathrm{GD}(\mathrm{Tb}=8$ ${ }^{\circ} \mathrm{C}$ ) para alcanzar la floración (Vallone et al., 2003).

\section{Procedimiento de análisis}

Se establecieron 10 fechas de siembra tentativas, y para cada una se obtuvo la suma de GD de las respectivas etapas de desarrollo (subperíodos) durante 23 años (entre 1968 y 1991). Esto permitió analizar la variabilidad de GD acumulados en cada uno de los subperíodos del modelo fenológico. Las fechas de siembra simuladas, que representan un rango potencial desde aquellas más tempranas hasta las más tardías, corresponden a los días 1 y 15 de cada mes entre agosto y diciembre. La verificación de la rutina fenológica, en particular respecto al subperíodo reproductivo, se realizó comparando su desempeño con la ocurrencia de la fase de floración que presentan los cultivares de uso en la región (Vallone et al., 2003).

Para estimar el rendimiento departamental de maíz se elaboró un modelo empírico que permite ponderar la influencia particular de cada etapa fenológica, sobre la base de la siguiente expresión (Unganai y Kogan, 1998):

$$
R_{e s t}=a+b\left(\sum_{\mathrm{j}=1}^{\mathrm{n}}\left(w_{j} \times N D_{j}\right)\right)
$$

donde $R_{e s t}$ es el rendimiento departamental estimado; $a$ y $b$ son coeficientes de ajuste obtenidos por regresión; ND es el valor promedio departamental del NDVI de cada subperíodo $\mathrm{j} ; w$ es un coeficiente de ponderación para cada subperíodo j, que se obtiene con la siguiente expresión:

$$
w_{j}=\frac{R_{j}^{2}}{\sum_{\mathrm{j}=1}^{\mathrm{n}} R_{j}^{2}}
$$

donde $\mathrm{R}^{2}$ es el coeficiente de determinación de cada subperíodo j; y el divisor es la suma de los coeficientes de determinación de los subperíodos considerados.

\section{RESULTADOS Y DISCUSIÓN}

\section{Evaluación del comportamiento fenológico esti- mado del maíz}

La duración estimada del ciclo de cultivo del maíz, la fecha de ocurrencia de la etapa de floración, como así también las condiciones térmicas que las acompañan para cada fecha de siembra en Marcos Juárez, se presentan en el Cuadro 1.

De acuerdo a los requerimientos estimados de sumas térmicas correspondientes a cada fecha de siembra potencial, hubo una variabilidad interanual reducida en las variables relacionadas con el comportamiento fenológico del cultivo, que se pone de manifiesto por los valores de desviación estándar, en todos los casos inferiores a $8 \%$ del valor promedio correspondiente. Mientras que para una misma fecha de siembra las sumas de temperatura o la duración de las etapas de desarrollo no se modifican notablemente de un año a otro, las diferencias son de mayor magnitud cuando se comparan las distintas fechas de siembra tentativas. De aquí se desprende que los valores medios de la duración de las etapas de desarrollo para cada fecha de siembra pueden ser utilizados en las diferentes campañas o ciclos sin cometer con ello errores muy apreciables. Además, se debe tener en cuenta que en este estudio, las imágenes procesadas corresponden a valores de NDVI compuestos de 10 días, lo que limita la precisión del análisis.

La Figura 2 se presenta a fin de verificar de manera gráfica el comportamiento de la rutina fenológica que se utiliza en la evaluación, especialmente durante la etapa reproductiva por su importancia sobre el rendimiento del cultivo de maíz (Dale y Daniels, 1995). Al resultado de la época del año en que transcurre el subperíodo reproductivo de acuerdo a las estimaciones del modelo, se superpuso la fecha estimada en que sucede la floración de los cultivares de uso en la región.

De esta forma, la ocurrencia estimada de la fase de floración de los cultivares más difundidos en Marcos Juárez, para todas las fechas de siembra, se cumple en un momento aproximadamente central 
Cuadro 1. Extensión y temperatura media del ciclo (T ciclo), subperíodo desde siembra a floración (DDS-F) y temperatura de la etapa (T S-F), y grados días acumulados (temperatura base $8^{\circ} \mathrm{C}$ ) (STT8) de 23 ciclos entre 1968 y 1991, para distintas fechas de siembra tentativas en Marcos Juárez, Córdoba, Argentina. Valores promedio (media) y desviación estándar (DE).

Table 1. Length and mean temperature of the life cycle ( $\mathrm{T}$ cycle), period from sowing date to flowering (DDS-F) and its temperature (T S-F) and growing degree days (base temperature $8{ }^{\circ} \mathrm{C}$ ) (STT8) of 23 cycles between 1968 and 1991, for different sowing dates in Marcos Juárez, Córdoba, Argentina. Average (media) and standard deviation (DE) values.

\begin{tabular}{llrrrrr}
\hline $\begin{array}{c}\text { Fecha de siembra } \\
\text { (Día juliano) }\end{array}$ & Media & $\begin{array}{c}\text { Extensión } \\
\text { (días) }\end{array}$ & $\begin{array}{c}\text { T ciclo } \\
\left({ }^{\mathbf{C}} \mathbf{C}\right)\end{array}$ & $\begin{array}{r}\text { DDS-F } \\
(\text { dí́as) }\end{array}$ & $\begin{array}{r}\text { T S-F } \\
\left({ }^{\mathbf{C}} \mathbf{C}\right)\end{array}$ & $\begin{array}{r}\text { STT8 } \\
\left({ }^{\circ} \mathbf{C} \text { días }\right)\end{array}$ \\
\hline 1 de agosto & 172 & 18,5 & 110 & 15,8 & $1.804,9$ \\
213 & DE & 6 & 0,4 & 6 & 0,4 & 35,9 \\
15 de agosto & Media & 162 & 19,2 & 100 & 16,5 & $1.822,9$ \\
227 & DE & 5 & 0,5 & 5 & 0,4 & 32,4 \\
1 de septiembre & Media & 152 & 20,2 & 88 & 17,6 & $1.850,7$ \\
244 & DE & 5 & 0,5 & 5 & 0,5 & 34,0 \\
15 de septiembre & Media & 144 & 21,0 & 80 & 18,6 & $1.869,4$ \\
258 & DE & 5 & 0,6 & 4 & 0,5 & 30,5 \\
1 de octubre & Media & 137 & 21,8 & 72 & 19,9 & $1.880,1$ \\
274 & DE & 6 & 0,7 & 4 & 0,6 & 32,6 \\
15 de octubre & Media & 131 & 22,4 & 65 & 21,0 & $1.886,0$ \\
288 & DE & 5 & 0,7 & 3 & 0,7 & 32,1 \\
1 de noviembre & Media & 126 & 23,0 & 60 & 22,2 & $1.889,1$ \\
305 & DE & 6 & 0,9 & 4 & 0,9 & 33,6 \\
15 de noviembre & Media & 124 & 23,3 & 56 & 23,4 & $1.885,7$ \\
319 & DE & 6 & 0,9 & 4 & 1,0 & 33,8 \\
1 de diciembre & Media & 126 & 22,9 & 53 & 24,2 & 1876,8 \\
335 & DE & 7 & 0,9 & 4 & 1,1 & 31,3 \\
15 de diciembre & Media & 132 & 22,2 & 52 & 24,5 & $1.868,6$ \\
349 & DE & 8 & 1,1 & 4 & 1,3 & 32,8 \\
\hline
\end{tabular}

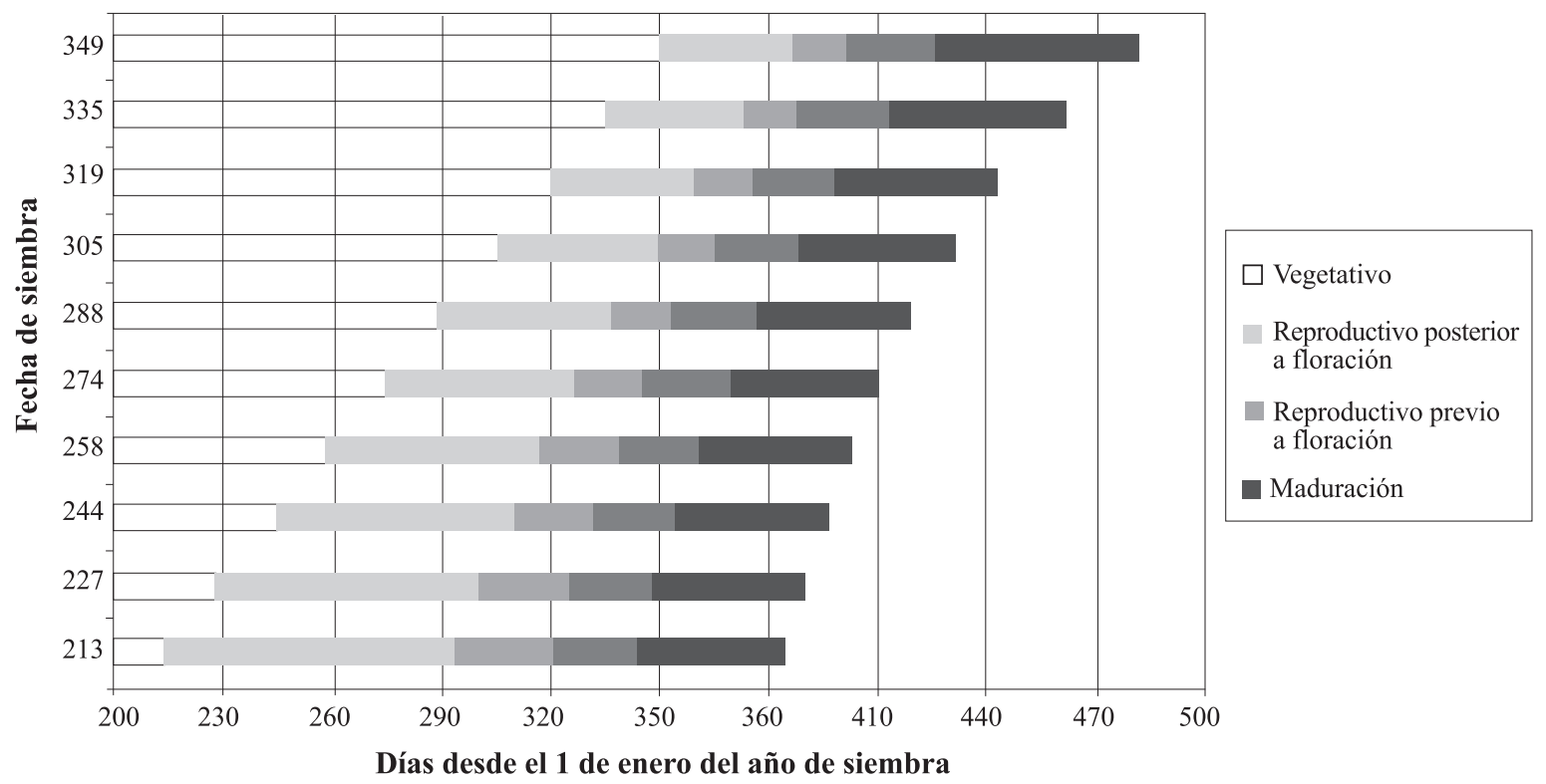

Figura 2. Fecha media estimada de los subperíodos del cultivo del maíz correspondiente a 23 ciclos y fecha de floración calculada con un valor de 850 grados días (temperatura base $=8{ }^{\circ} \mathrm{C}$ ), con respecto a la fecha de siembra (día $213=1$ de agosto,..., día $349=15$ de diciembre).

Figure 2. Estimated mean date of corn development periods of 23 cycles and flowering date calculated with 850 degree days (base temperature $=8^{\circ} \mathrm{C}$ ), in relation to sowing date (day $213=1$ August,..., day $349=15$ December). 
del subperíodo reproductivo (Figura 2), que es determinado por el modelo fenológico utilizado para discriminar las etapas de desarrollo del maíz. Asimismo, se manifiesta el acortamiento de la etapa vegetativa a medida que la fecha de siembra se retrasa (Warrington y Kanemasu, 1983), alcanzando en las siembras tardías de diciembre una extensión inferior al 50\% respecto a las siembras más tempranas de agosto, cuando esta etapa se prolonga entre 70 y 80 días. En cuanto al tiempo a floración, para las fechas del 1 y del 15 de diciembre se estimaron 53 y 52 días, respectivamente. Estos valores son similares a los observados en Marcos Juárez para las fechas de siembra más tardías, que es de 49 días para los cultivares de ciclo corto-intermedio y de 54 días para los de ciclo largo (Vallone et al., 2003).
Relación entre el rendimiento departamental de maíz y el NDVI durante cada etapa de desarrollo del cultivo

Hayes y Decker (1996) en el cinturón del maíz de EE.UU., Mika et al. (2002) en Hungría, y Mkhabela et al. (2005) en Swazilandia, han mostrado que los datos del NOAA-AVHRR pueden ser utilizados para explicar una parte sustancial de la variabilidad del rendimiento de maíz sobre grandes extensiones geográficas. En este mismo sentido, se estableció la relación entre el rendimiento estimado de maíz y el valor de NDVI promedio del Departamento Marcos Juárez, con el propósito de discriminar la influencia de cada etapa de desarrollo en la productividad de esta región. El análisis de correlación, en general, expresa una asociación positiva entre el NDVI acumulado en cada etapa y el rendimiento de maíz (Figura 3).

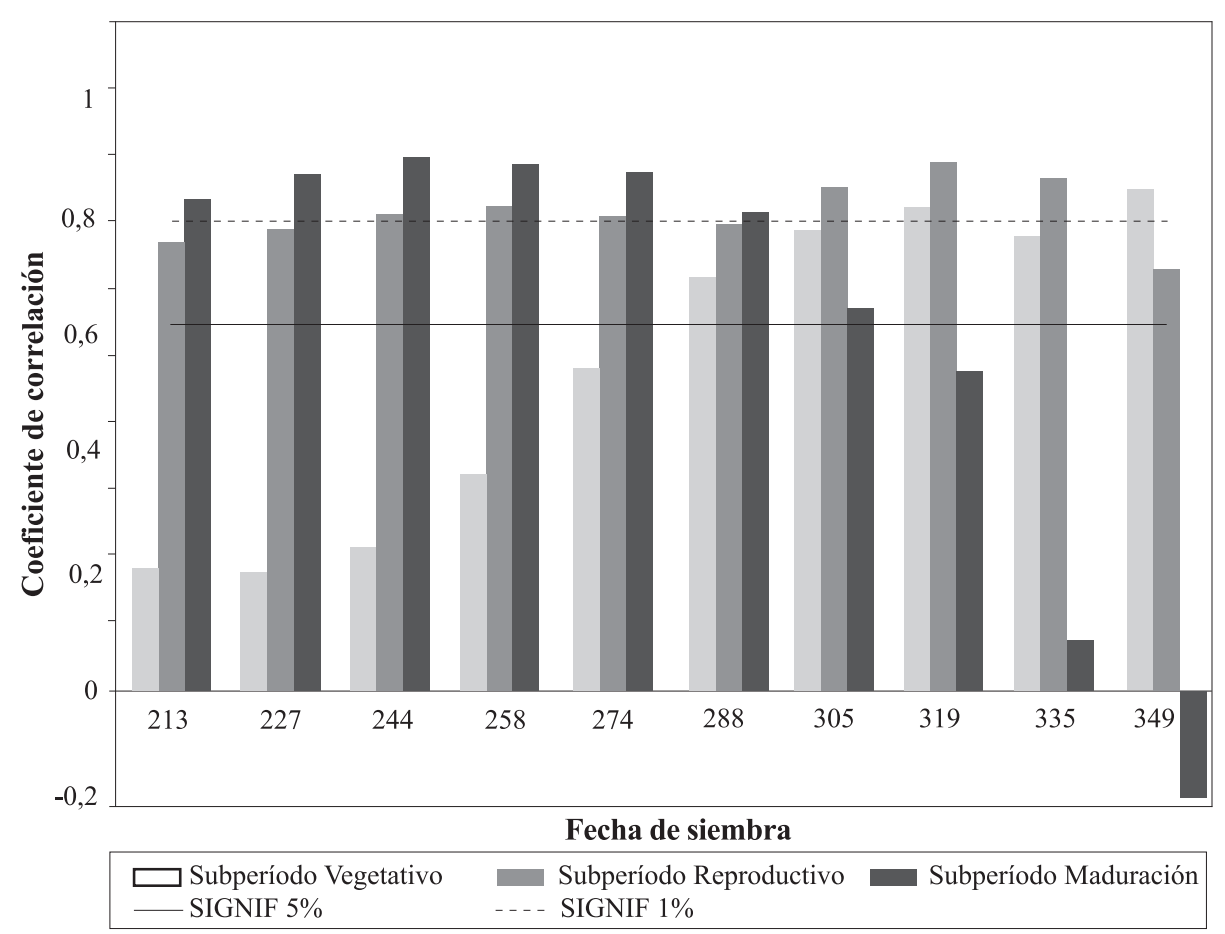

Figura 3. Coeficientes de correlación entre el rendimiento de maíz y el Î́ndice de Vegetación de la Diferencia Normalizada)(NDVI) del Departamento Marcos Juárez en distintas etapas del cultivo y diferentes fechas de siembra. Se señala también el nivel de los casos significativos al 1 y $5 \%$.

Figure 3. Correlation coefficients between corn yield and Normalized Difference Vegetation Index (NDVI) of Marcos Juárez Department in different development stages and sowing dates. Significant cases of 1 and $5 \%$ are also shown. 
Cuadro 2. Coeficientes de regresión (a: intercepción y b: pendiente) y determinación $\left(R^{2}\right)$ de los modelos de estimación del rendimiento para cada fecha de siembra considerando el efecto sólo de los subperíodos que presentan influencia significativa $(P<0,05)$ (Ver Figura 3).

Table 2. Regression (a: interception and b: slope) and determination $\left(\mathbf{R}^{2}\right)$ coefficients of yield estimation models for each sowing date considering only periods highly significant $(P<0.05)$ (See Figure 3).

\begin{tabular}{lrrrrrrrrrr}
\hline & \multicolumn{10}{c}{ Fecha de siembra (día del año) } \\
\cline { 2 - 11 } & \multicolumn{1}{c}{$\mathbf{2 1 3}$} & $\mathbf{2 2 7}$ & $\mathbf{2 4 4}$ & $\mathbf{2 5 8}$ & $\mathbf{2 7 4}$ & $\mathbf{2 8 8}$ & \multicolumn{1}{c}{$\mathbf{3 0 5}$} & $\mathbf{3 1 9}$ & $\mathbf{3 3 5}$ & $\mathbf{3 4 9}$ \\
\hline $\mathrm{b}$ & $18.728,9$ & $17.603,7$ & $16.629,4$ & $15.866,4$ & $16.629,4$ & $18.815,0$ & $16.951,0$ & $16.120,2$ & $14.691,6$ & $14.497,4$ \\
$\mathrm{a}$ & $-5.917,6$ & $-5.451,2$ & $-5.073,0$ & $-4.798,0$ & $-5.073,0$ & $-6.273,6$ & $-5.139,7$ & $-4.798,6$ & $-4.431,8$ & $-4.530,2$ \\
$\mathrm{R}^{2}$ & 0,81 & 0,83 & 0,84 & 0,82 & 0,84 & 0,79 & 0,79 & 0,82 & 0,75 & 0,69 \\
\hline
\end{tabular}

La correlación entre el NDVI de cada etapa de desarrollo y la productividad global del departamento cambia a medida que son consideradas diferentes fechas de siembra (Figura 3). De todos modos, la etapa reproductiva, para cualquier fecha de siembra, expresa siempre una elevada asociación con el rendimiento, alcanzando valores de correlación de carácter significativo en todos los casos al nivel de $5 \% \mathrm{y}$, en algunas fechas superiores a $1 \%$. Este comportamiento se explica por cuanto en la etapa reproductiva ocurre la floración, momento en que el maíz tiene mayor sensibilidad al estrés hídrico (Dale y Daniels, 1995; Sadrás y Calviño, 2001). En las fechas de siembra tempranas, el subperíodo de maduración también tiene una alta capacidad para explicar la variabilidad, en tanto en las fechas tardías es la etapa vegetativa la que exhibe esta mayor capacidad. El análisis conjunto de las Figuras 2 y 3 revela que la fecha de siembra del 15 de octubre (día 288), con su ciclo comprendido aproximadamente entre los días 290 y 410 , acusa correlación significativa en todas las etapas de desarrollo, probablemente como consecuencia de ser la fecha de siembra más difundida en la región. Esta información puede ser útil a fin de considerar una estrategia para elaborar algún sistema de pronóstico de rendimiento para la región que considere la evolución del área sembrada conforme avanza la estación.

\section{Modelo de estimación}

Se desarrolló un modelo empírico de estimación del rendimiento departamental de maíz, ponderando el efecto parcial del NDVI sobre el rendimiento durante cada etapa fenológica. Unganai y Kogan
(1998) utilizaron este procedimiento para una región del SE de Africa, considerando como variables independientes sólo el NDVI de los períodos semanales que presentaban correlación significativa. El denominador del coeficiente de ponderación $w_{j}$ se obtuvo de la suma de $R_{j}^{2}$ de estas etapas de desarrollo. Los coeficientes de correlación y regresión de los modelos obtenidos para cada fecha de siembra se presentan en el Cuadro 2 .

A partir de estos modelos, que constituyen expresiones relativamente simples, se obtienen estimaciones que explican alrededor de $80 \%$ de la variabilidad interanual del rendimiento departamental. De acuerdo a los resultados que se obtienen para las siembras tempranas del 1 de septiembre (244) y del 1 de octubre (274), cuando en ambos casos se alcanza un $\mathrm{R}^{2}$ máximo de 0,84 , sería factible elaborar una proyección anticipada del rendimiento desde finales de enero y mediados de febrero, es decir un par de meses antes de la cosecha. Este análisis coincide en términos de anticipo con los resultados obtenidos por Mkhabela et al. (2005).

Los resultados también confirman que a los efectos de pronosticar el rendimiento de maíz por medios satelitales es conveniente emplear distintas fechas de muestreo (análisis multitemporal) durante el ciclo (Chang et al., 2003). Resultados de Shanahan et al. (2001), quienes emplearon el índice GNDVI para correlacionar con el rendimiento de maíz, obtuvieron coeficientes de 0,7 y 0,92 en las dos campañas que analizaron, y los valores más altos los obtuvieron durante la etapa del llenado de granos. 


\section{CONCLUSIONES}

El esquema de etapas fenológicas utilizado ofrece una delimitación confiable del calendario del cultivo de maíz en el Departamento Marcos Juárez, en particular con respecto a la ocurrencia estimada de la etapa reproductiva. La variabilidad interanual que presenta la duración de los distintos subperíodos es reducida con respecto a las diferencias que acontecen entre fechas de siembra. En cualquier fecha de siembra tentativa, el valor de NDVI durante la etapa reproductiva expresa siempre una elevada asociación con el rendimiento. En las fechas de siembra tempranas, el NDVI acumulado durante la etapa de maduración también tiene una alta capacidad para explicar la variabilidad del rendimiento; mientras que en las tardías es la etapa vegetativa, en cambio, la que exhibe esta mayor capacidad. Un modelo desarrollado con esta información permite estimar con buena precisión el rendimiento de maíz desde finales de enero y febrero, lo que se anticipa en un par de meses a la cosecha.

\section{LITERATURA CITADA}

Agbu, P.A., and M.E. James. 1994. The NOAA/NASA Pathfinder AVHRR Land Data Set. User's Manual. 104 p. Goddard Distributed Active Archive Center, NASA, Goddard Space Flight Center, Greenbelt, Maryland, USA.

Barron, J., J. Rockstrom, F. Gichuki, and N. Hatibu. 2003. Dry spell analysis and maize yields for two semi-arid locations in East Africa. Agric. For. Meteorol. 117:2337.

Brown, D.M., and A. Bootsma. 1993. Crop heat units for corn and other warm season crops in Ontario. Ont. Minist. Agric. Food. Factsheet, Agdex 111/31. ISSN $\mathrm{N}^{\circ}$ 0225-7882. Ontario Ministry of Agriculture and Food, Ontario, Canadá.

Burt, J.E., J.T. Hayes, P.A. O'Rourke, W.H. Terjung, and P.E. Todhunter. 1981. A parametric crop water use model. Water Resour. Res. 17:1095-1108.

Chang, J., D.E. Clay, K. Dalsted, S. Clay, and M. O'Neill. 2003. Corn (Zea mays L.) yield prediction using multispectral and multidate reflectance. Agron. J. 95:1447-1453.

Dale, R.F., and J.A. Daniels. 1995. A weather-soil variable for estimating soil moisture stress and corn yield probabilities. Agron. J. 87:1115-1121.

Dawbin, K.W., J.C. Evans, M.J. Duggin, and E.K. Leggett. 1980. Classification of wheat areas and prediction of yields in North-western New South Wales by repetitive Landsat data. Aust. J. Agric. Res. 31:449-453.

de la Casa, A.C. 1992. Modelo estadístico de pronóstico de rendimiento de maíz para la región semiárida de Córdoba basado en datos pluviométricos areales. Agriscientia (Córdoba, Argentina) 9(2):87-96.
Doorembos, J., y A.H. Kassam. 1979. Efectos del agua sobre el rendimiento de los cultivos. Estudio FAO: Riego y Drenaje No 33. 212 p. FAO, Roma, Italia.

Doraiswamy, P.C., S. Moulin, P.W. Cook, and A. Stern. 2003. Crop yield assessment from remote sensing. Photogramm. Eng. Remote Sens. 69(6):665-674.

Hayes, M.J., and W.L. Decker. 1996. Using NOAA AVHRR data to estimate maize production in the United States corn belt. Int. J. Remote Sens. 17:31893200.

Holben, B.N. 1986. Characteristics of maximum-value composite images from temporal AVHRR data. Int. J. Remote Sens. 7:1417-1434.

Kiniry, J.R., and R. Bonhomme. 1991. Predicting maize phenology. p. 115-132. In Hodges, T. (ed.) Predicting crop phenology. CRC Press, Boca Raton, Florida, USA.

Lobell, D.B., G.P. Asner, J.I. Ortiz-Monasterio, and T.L. Benning. 2003. Remote sensing of regional crop production in the Yaqui Valley, Mexico: estimates and uncertainties. Agric. Ecosyst. Environ. 94:205-220.

Maas, S.J. 1988. Using satellite data to improve model estimates of crop yield. Agron. J. 80:662-665.

Maas, S.J. 1993. Within-season calibration of modeled wheat growth using remote sensing and field sampling. Agron. J. 85:669-672.

Meyer, S.J., K.G. Hubbard, and D.A. Wilhite. 1993a. A crop-specific drought index for corn. I. Model development and validation. Agron. J. 85:388-395.

Meyer, S.J., K.G. Hubbard, and D.A. Wilhite. 1993b. A crop-specific drought index for corn. II. Application in drought monitoring and assessment. Agron. J. 85:396-399. 
Mika, J., J. Kerényi, A. Rimóczi-Paál, Á. Merza, C. Szinell, and I. Csiszár. 2002. On correlation of maize and wheat yield with NDVI: Example of Hungary (1985-1998). Adv. Space Res. 30:2399-2404.

Mkhabela, Ma. S., Mi. S. Mkhabela, and N.N. Mashinini. 2005. Early maize yield forecasting in four agroecological regions of Swaziland using NDVI data derived from NOAA's-AVHRR. Agric. For. Meteorol. 129:1-9.

Nielsen, R.L., P.R. Thomison, G.A. Brown, A.L. Halter, J. Wells, and K.L. Wuethrich. 2002. Delayed planting effects on flowering and grain maturation of dent corn. Agron. J. 94:549-558.

Ritchie, J.T., and D.S. Nesmith. 1991. Temperature and crop development. p. 5-29. In J. Hanks, and J.T. Ritchie (eds.) Modeling plant and soil systems. Agron. Monogr. 31. ASA, CSSA and SSSA, Madison, Wisconsin, USA.

Sadrás, V.O., and P.A. Calviño. 2001. Quantification of grain yield response to soil depth in soybean, maize, sunflower, and wheat. Agron. J. 93:577-583.

SAGyA. 2002. Estadísticas de producción agrícola. Secretaría de Agricultura, Ganadería y Alimentos de la Provincia de Córdoba. 2002. Disponible en http:// www.cba.gov.ar Leído 12 diciembre 2003.

Shanahan, J.F., J.S. Schepers, D.D. Francis, G.E. Varvel, W.W. Wilhelm, J.M. Tringe et al. 2001. Use of remotesensing imagery to estimate corn grain yield. Agron. J. 93:583-589.

Smith, R.C.G., J. Adams, D.J. Stephens, and P.T. Hick. 1995. Forecasting wheat yield in a Mediterraneantype environment from the NOAA satellite. Aust. J. Agric. Res. 46:113-125.
Steinwand, D.R., J.A. Hutchinson, and J.P. Snyder. 1992. Map projections from global and continental data sets, and an analysis of distortion caused by reprojection. U.S. Geological Survey/Center for Earth Resources Observation and Science contract report. EROS Data Center, Sioux Falls, South Dakota, USA.

Stewart, D.W., L.M. Dwyer, and L.L. Carrigan. 1998. Phenological temperature response of maize. Agron. J. 90:73-79.

Tarpley, J., S. Schneider, and R. Money. 1984. Global vegetation indices from NOAA-7 meteorological satellite. J. Clim. Appl. Meteorol. 23:491-494.

Unganai, L., and F. Kogan. 1998. Drought monitoring and corn yield estimation in Southern Africa from AVHRR data. Remote Sens. Environ. 63:219-232.

Vallone, P., V. Gudelj, C. Galarza, y B. Masiero. 2003. Ensayo comparativo de rendimiento de maíz de segunda. Campaña 2002/2003. 3 p. Documentos EE Marcos Juárez, INTA. Marcos Juárez, Córdoba, Argentina.

Warrington, I.J., and E.T. Kanemasu. 1983. Corn growth response to temperature and photoperiod. I. Seeding emergence, tassel initiation, and anthesis. Agron. J. 75:749-754.

Weiss, M., D. Troufleau, F. Baret, H. Chauki, L. Prévot, A. Olioso, et al. 2001. Coupling canopy functioning and radiative transfer models for remote sensing data assimilation. Agric. For. Meteorol. 108:113-128.

WMO. 1983. Guide to climatological practices. $198 \mathrm{p}$. $2^{\text {nd }}$ ed. World Meteorological Organization, Geneva, Switzerland. 